\title{
Binding of the transcription factor Slug to the L1CAM promoter is essential for transforming growth factor- $\$ 1$ (TGF-B)-induced L1CAM expression in human pancreatic ductal adenocarcinoma cells
}

\author{
CLAUDIA GEISMANN $^{1}$, ALEXANDER ARLT ${ }^{1}$, IRIS BAUER ${ }^{1}$, MARCO PFEIFER $^{2}$, UWE SCHIRMER ${ }^{2}$, \\ PETER ALTEVOGT ${ }^{2}$, SUSANNE SEBENS MÜERKÖSTER ${ }^{1,3}$ and HEINER SCHÄFER ${ }^{1}$
}

\begin{abstract}
${ }^{1}$ Department of Internal Medicine 1, Laboratory of Molecular Gastroenterology \& Hepatology, UKSH-Campus Kiel, ArnoldHeller-Strasse 3, Bldg. 6, 24105 Kiel; ${ }^{2}$ German Cancer Research Center, Division of Translational Immunology D015, Im Neuenheimer Feld 280, 69120 Heidelberg; ${ }^{3}$ Institute of Experimental Medicine, UKSH-Campus Kiel, 24105 Kiel, Germany
\end{abstract}

Received August 9, 2010; Accepted September 24, 2010

DOI: 10.3892/ijo_00000846

\begin{abstract}
Members of the Slug/Snail family of transcription factors are thought to drive epithelial-mesenchymal-transition (EMT) in preneoplastic epithelial cells, thereby contributing to malignant transformation. One mediator in the EMT of pancreatic ductal adenocarcinoma (PDAC) cells and a potential target gene of Slug is the cellular adhesion molecule L1CAM. Using the human pancreatic ductal epithelial cell line H6c7 and the PDAC cell line Panc1, we could show that along with TGF-ß1-induced EMT, L1CAM expression is increased in a Slug- but not Snail-dependent fashion. Two E-box recognition motifs in the L1CAM promoter upstream of the most distal transcriptional start site could be verified by gel shift and supershift assay to interact with Slug. ChIP assays detected an increased interaction of Slug with both recognition motifs of the human L1CAM promoter in TGF-ß1treated H6c7 cells, whereas binding of Snail was downregulated. Moreover, ChIP assays with Panc1 cells confirmed this interaction of Slug with the human L1CAM promoter and further detected an interaction of both recognition sites with RNA-polymerase II in a Slug-dependent fashion. Luciferase reporter gene assays using wild-type or singleand double-mutated variants of the L1CAM promoter confirmed transcriptional activation by Slug involving both
\end{abstract}

Correspondence to: Dr Heiner Schäfer, Department of Internal Medicine 1, Laboratory of Molecular Gastroenterology \& Hepatology, UKSH-Campus Kiel, Arnold-Heller-Strasse 3, Bldg. 6, 24105 Kiel, Germany

E-mail: hschaef@1med.uni-kiel.de

Abbreviations: ChIP, chromatin immunoprecipitation; EMT, epithelial-mesenchymal-transition; PDAC, pancreatic ductal adenocarcinoma; TGF- $\beta 1$, transforming growth factor- $\$ 1$

Key words: epithelial-mesenchymal transition, tumorigenesis, transcription factor, gene expression recognition motifs. By demonstrating the direct transcriptional control of L1CAM expression through Slug during TGF-ß1-induced EMT of PDAC cells, our findings point to a novel mechanism by which Slug contributes quite early to tumorigenesis. Moreover, our study is the first one describing the control of the human L1CAM promoter in tumor cells.

\section{Introduction}

Pancreatic ductal adenocarcinoma (PDAC) is a highly malignant type of tumor associated with rapid tumor progression and early metastasis (1). Tumorigenesis of PDAC presumably originates from ductal epithelial cells and is crucially driven by interactions with pancreatic myofibroblasts, the main compartment of fibrosis in chronic pancreatitis and the tumoral stroma. It was shown, that heterotypic signals released by mesenchymal cells in the stromal compartment of normal or neoplastic tissues are responsible for the induction of epithelial- mesenchymal-transition (EMT) in epithelial cells (2). In the process of EMT, epithelial characteristics disappear and the cells adopt a mesenchymal phenotype, closely correlated with enhanced motility and invasive predisposition $(3,4)$. One of the best investigated inducers of EMT are the members of the transforming growth factor- $\beta$ (TGF-ß) family (2). It is well established that TGF- 11 acts as a suppressor in quite early stages of tumor development but also boosts angiogenesis and metastatic growth in advanced stages of tumorigenesis (5), thus pointing to a dual role of TGF- $\$ 1$ in tumorigenesis. Moreover, previous studies clearly demonstrated that TGF- $\beta 1$, besides its growth suppressive abilities in early tumorigenesis, already exerts several other effects such as promoting EMT and migration $(6,7)$ resulting in a net-effect of TGF- 31 that is rather tumor promoting than tumor suppressive.

TGF-ß1 can mediate its effects via different signaling cascades, e.g. involving activation of smad proteins or members of the MAP kinases. Moreover, TGF- $\beta 1$ has been shown to induce members of the Snail/Slug transcription factor family which have also been implicated in promoting EMT in systems 
of development and carcinogenesis through binding to the specific recognition motif CAGGTG (8). In many types of cancer these proteins lead to the downregulation of E-cadherin by transcriptional repression, resulting in a disassembly of adhered junctions (9-12). Furthermore, Snail can act as transcriptional inducer, e.g. of genes encoding vimentin, matrix metalloproteinases or ZEB-1 $(13,14)$. In tissues of PDAC, elevated expression of Slug compared to normal surrounding parenchyma was shown but, most notably, no obvious correlation between Slug and E-cadherin was found (15).

Recently, it was demonstrated that TGF- $\beta 1$ induces the expression of the promigratory molecule L1CAM (L1/CD171) in human pancreatic ductal epithelial cells (16). L1CAM is a 200-220-kD glycoprotein and a member of the immunoglobulin superfamily. This type- 1 transmembrane protein contains an ectodomain consisting of six immunoglobulinlike domains and five fibronectin type III homologous repeats linked via a single transmembrane sequence to a conserved cytoplasmic domain (17). The L1CAM gene encompasses 29 exons, the first one (sometimes denoted exon- 0 ) consists of the 5'-UTR and the second one (sometimes denoted exon-1) contains the start codon. Beside six different transcriptional start sites located upstream of exon-1, various splicing variants of L1CAM are known, including two major products encoding for the 1257 and 1253 amino acid proteins. L1CAM plays an important role in the nervous system development, including neuronal migration and differentiation (18). L1CAM can interact with itself (homophilic) but also with a variety of heterophilic ligands such as integrins, CD24, neurocan, neuropilin-1 and other members of the neural cell adhesion family. Besides L1CAM expression in neuronal cells, overexpression of L1CAM is observed in various cancer entities such as melanoma, ovarian cancer, colon cancer, and PDAC (19-22) and the expression of L1CAM correlates with poor prognosis and short survival times. Furthermore, it was noted that L1CAM can be associated with cell growth, cell migration and chemoresistance in tumor cells (20,23-26).

As we recently showed, these L1CAM-mediated alterations are induced quite early in the pancreatic ductal epithelial cell line H6c7 subjected to TGF-B1-induced EMT. This cell line has been immortalized by human papilloma virus-16 $\mathrm{H} 6 \mathrm{H} 7$ gene transfection, but largely retained an epithelial phenotype (e.g. non-migratory, no vimentin expression, normal E-cadherin expression) and is, most importantly, not tumorigenic. Thus, H6c7 cells are commonly used as cellular tool to investigate a pancreatic duct-like cellular phenotype (27). Interestingly, the increased expression of L1CAM in H6c 7 cells treated with TGF- 11 depends on Slug, but not on Snail (16), pointing to a particular role of Slug during EMT of the pancreatic duct epithelium (15). In support of this assumption, the strong L1CAM expression in the PDAC cell line Panc1 which is TGF- $\$ 1$ responsive in an autocrine fashion and which exhibits a pronounced mesenchymal phenotype (e.g. significant migratory potential, strong vimentin and reduced E-cadherin expression) was shown to be also dependent on Slug (16).

The aim of the present study was therefore to provide experimental proof that Slug induces L1CAM expression by a direct interaction with the human L1CAM promoter. Using H6c7 cells subject of TGF- 11 treatment on the one hand and the TGF- $ß 1$ secreting PDAC cell line Panc1 on the other hand we demonstrate for the first time, that the promoter of the human L1CAM gene is regulated by Slug, an action related to the EMT of cancer cells.

\section{Materials and methods}

Cell lines and materials. The human pancreatic ductal epithelial cell line H6c7 (kindly provided by Professor M.S. Tsao, Ontario Cancer Center, Toronto, Canada), the PDAC cell line Panc1 (donated by $\mathrm{H}$. Kalthoff, Institute of Experimental Cancer Research, UKSH-Campus Kiel, Kiel, Germany) and HeLa cells were cultured as reported $(16,28)$. For transfection and knock-down experiments, cells were seeded into six-well plates at a density of $2-3 \times 10^{5}$ cells/well or into twelve-well plates at a density of $1 \times 10^{5}$ cells/well. In addition, H6c7 cells cultured on six-well plates and used for ChIP assays were treated with TGF- 11 (R\&D-Systems, Wiesbaden, Germany) at $10 \mathrm{ng} / \mathrm{ml}$.

Knock-down experiments. For siRNA transfection, medium was removed and fresh medium supplied with $12 \mu 1$ HiperFect (Qiagen, Hilden, Germany) and $150 \mathrm{ng} /$ well Slug-specific siRNA (SI 00047537), Snail-specific siRNA (SI 02636424) or negative control siRNA (all from Qiagen) were added. As determined by transfection with a FITC-labeled siRNA, the transfection efficacy of siRNA was $60-70 \%$ in $\mathrm{H} 6 \mathrm{c} 7$ and Panc1 cells.

Cell transfection. For gel shift assay and Western blotting, $2 \times 10^{5} \mathrm{HeLa}$ or H6c7 cells were seeded into six-well plates and transfected with an empty vector (pcDNA3.1) or pcDNA3.1Slug $(0,6 \mu \mathrm{g} /$ well $)$ using the Effectene transfection reagent (Qiagen) following the manufacturer's instructions. A transfection efficacy of $\sim 30 \%$ (Panc1) and 15\% (H6c7) was yielded by GFP control transfection.

Western blotting. Preparation of whole-cell lysates and nuclear extracts as well as electrophoresis was performed as described (28). For immunoblotting, L1CAM antibodies were obtained from Acris Antibodies (Hiddenhausen, Germany), and Snail and Slug antibodies (sc-10436X and sc-10432X, respectively) from Santa Cruz Biotechnology (Heidelberg, Germany). The antibody against Hsp90 was purchased from Santa Cruz Biotechnology, as well, and the tubulin antibody was from Sigma (Deisenhofen, Germany). Primary antibodies were incubated overnight at $4^{\circ} \mathrm{C}$ at $1: 1000$ or 1:2000 dilution in TBST (50 mM Tris/HCl, $\mathrm{pH} \mathrm{7.6,} \mathrm{and} 150 \mathrm{mM} \mathrm{NaCl}$, $0.05 \% \mathrm{v} / \mathrm{v}$ Tween-20) supplemented with $5 \%(\mathrm{w} / \mathrm{v})$ non-fat milk powder. After extensive washing with $0.05 \%$ Tween-20 in TBS, blots were exposed to the appropriate horseradish peroxidase-conjugated secondary antibodies (Cell Signaling Technology) at 1:1000 dilution for $1 \mathrm{~h}$ at room temperature. After washing in TBST, blots were developed with SuperSignal West Dura Extended Duration Substrate (Perbio Sciences, Bonn, Germany).

RNA preparation, conventional RT-PCR and real-time PCR. Total RNA isolation from H6c7 and Panc1 cells cultured on six-well plates, reversed transcription of mRNA and real-time 
PCR was done as described previously $(16,29)$. The following primers were used: Slug, 5'-atattcggacccacacattacct-3' (forward) and 5'-gcaaatgctctgttgcagtga-3' (reverse); Snail, 5'-ctgctccac aagcaccaagagtc-3' (forward) and 5'-ccagctgcctccctccac-3' (reverse); L1CAM, 5'-tcacgggcaacaacagcaact-3' (forward) and 5'-cggcttcctgtcaatcatgct-3' (reverse). For normalization, B-actin was amplified with primers from Lonza (Verviers, Belgium). In order to detect the exon-1/exon-2 encoding L1CAM mRNA transcript, the primer pair 5'-agccegagcggtgg ccgca-3' (forward) and 5'-taccgcagcgccacgaccatc-3' (reverse) was used in a conventional RT-PCR experiment yielding a 153-bp product.

Gel shift assay. For gel shift assay, nuclear extracts were incubated with a $\gamma^{32} \mathrm{P}$-labeled oligonucleotide 3'-cgtcttcact CAGGTGcctagtcttg-5' (1st Slug site) or 3'-gcgttgcgtgCAG GTGcccatcgctg-5' (2nd Slug site) containing the putative Slug/Snail-binding sites. After incubation for $30 \mathrm{~min}$ at room temperature, samples were separated by gel electrophoresis at $12 \mathrm{~A}$ and $4^{\circ} \mathrm{C}$. Gels were dried and DNA-protein complexes were visualized by autoradiography using the Hyperfilm-MP (GE Healthcare, Freiburg, Germany). For supershift assays $4 \mu \mathrm{g}$ of Slug antibody (sc-10436X, Santa Cruz Biotechnology) were added to the nuclear extracts.

Chromatin immunoprecipitation assay (ChIP). A ChIP assay kit from Upstate Biotechnology (Schwalbach, Germany) was used following the manufacturer's instructions. DNA from H6c7 (TGF-ß1 and siRNA treated) or Panc1 cells (siRNA treated) was submitted to immunoprecipitation with $4 \mu \mathrm{g}$ of normal goat IgG, anti-Slug (sc-10436X, Santa Cruz Biotechnology), anti-Snail (sc-10432X, Santa Cruz Biotechnology), anti-RNA-polymerase II (sc-900, Santa Cruz Biotechnology) or normal rabbit IgG (Santa Cruz Biotechnology) (16). Primers used for real-time PCR: GAPDH 5'-agcgcaggcctcaagacctt-'3 (forward) and 5'-aagaagatgcggct gactgt-3' (reverse), 1st Slug site 5'-gaagatgcctgcaagtgccc-'3 (forward) and 5'-tggacccatctgtgctcagaga-'3 (reverse) and 2nd Slug site 5'-attcgggagaggtcccgggagt-3' (forward) and 5'-agga agggggatgctcccttagc-3' (reverse).

Generation of the human Slug expression vector. cDNA from H6c7 cells was submitted to PCR using the forward primer 5'-gatgccgcgctccttcct-3' and the reverse primer 5'-ggctgct gtgtagcacactagg-3' yielding a 809-bp amplicon encompassing the full length reading frame of human Slug. This amplicon was TA-cloned into the pCR3.1 vector (Invitrogen, Karlsruhe, Germany) and verified by DNA cycle sequencing.

Generation of LICAM promoter luciferase reporter gene constructs and site-directed mutagenesis. Using the forward primers 5'-accacagctcagtgcctcatgg-3' and the reverse primer 5'-gctagctgcggcagcagcggctg-3' creating a NheI restriction site, a 1425-bp fragment (position 39086-40511) of the 5 -flanking region of the human LCAM gene $\left(\right.$ GeneBank $^{\odot}$ accession U52112.2) with a reported transcription start at position 39109 (30) was amplified from human genomic DNA. After TA-cloning into the pCR2.1 vector (Invitrogen), the promoter fragment was subcloned into the pGL3 basic luciferase vector (Promega, Mannheim, Germany) via KpnI and NheI sites. For promoter constructs with mutated Slug/ Snail-binding sites, primers with a substitution of CAGGTG to TGGGTG in the 1st Slug-binding site and GTGGTG in the 2nd Slug-binding site were designed. Mutations were inserted by using QuickChange Site-directed mutagenesis kit (Stratagene, La Jolla, USA) according to manufacturer's instructions. Each promoter construct was cloned into the firefly luciferase reporter gene containing vector pGL3. All constructs were verified by automated BigDye (PerkinElmer, Waltham, USA) DNA cycle sequencing using an ABI 3770 instrument (Perkin-Elmer).

Dual luciferase reporter gene assay. For reporter gene assays, Panc1 and H6c7 cells were seeded into 12-well plates. Twenty-four hours after Slug siRNA treatment (Panc1) or pcDNA3.1-Slug transfection (H6c7), cells were transfected with promoterless pGL3 or one of the pGL3-L1CAM promoter constructs (all $0.4 \mu \mathrm{g} /$ well) together with a renilla luciferase construct (pRL-TK; $0.1 \mu \mathrm{g} /$ well) using Effectene transfection reagent. For lysate preparation, cells were washed with PBS and lysed in $150 \mu 1 /$ well passive lysis buffer (Promega). Lysates were centrifuged for $2 \mathrm{~min}$ at $4^{\circ} \mathrm{C}$, $13000 \mathrm{rpm}$. The supernatant $(20 \mu \mathrm{l})$ was used for the dual luciferase assay procedure, using Dual-Glow luciferase assay system from Promega. Firefly luciferase expression (L1CAM promoter constructs) was normalized to constitutive renilla luciferase expression.

Statistical analysis. Data are presented as mean \pm standard deviations. Statistical significance was determined by the paired student's t-test. $\mathrm{p}<0.05$ was considered statistically significant.

\section{Results}

L1CAM expression in H6c7 pancreatic ductal epithelial cells and in Pancl PDAC cells is Slug dependent. Since Snail and Slug recognize the same E-box binding motif and to substantiate our recent findings on the Slug dependency of the TGF-B1-induced L1CAM expression in the pancreatic ductal epithelial cell line H6c7 (16), we first analysed the effect of TGF- 31 on Slug and Snail expression in these cells. As shown by real-time PCR, treatment of H6c7 cells with TGF- $\$ 1$ resulted in an increase of Slug mRNA levels in a time-dependent fashion, with a maximal effect at 3 and $6 \mathrm{~h}$ after TGF-ß1 administration (Fig. 1A, left panel). Under the same conditions, mRNA level of Snail were more weakly altered after TGF- $\$ 1$ treatment of $\mathrm{H6c} 7$ cells (Fig. 1A, left panel). Western blot analysis confirmed the inducing effect of TGF- 11 on Slug expression, as shown by the elevated levels of Slug protein in nuclear extracts from H6c7 cells treated with TGF-B1 for 6-24 h (Fig. 1A, right panel). In contrast, Snail protein levels did not increase in $\mathrm{H} 6 \mathrm{c} 7$ cells after TGF-B1 treatment (Fig. 1A, right panel) but rather decreased slightly.

In order to validate that the TGF- $\beta 1$-induced L1CAM expression depends on Slug, the impact of siRNA-mediated knock-down of Snail and Slug on L1CAM expression was analysed. As shown by real-time PCR and Western blot (Fig. 1B and C), the knock-down of Slug expression clearly 
A)

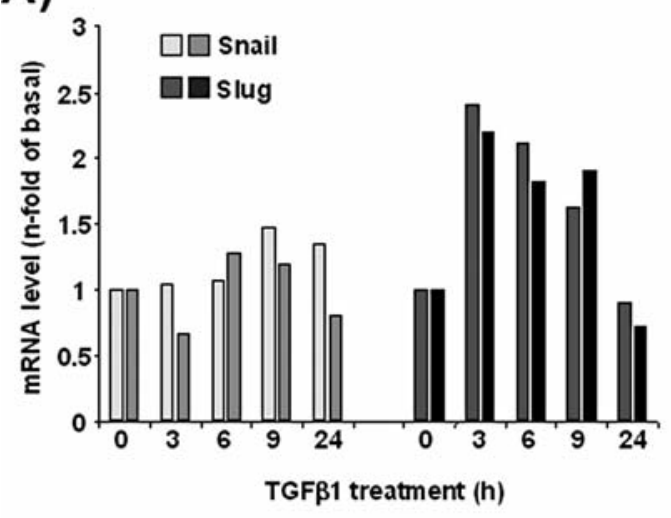

H6c7

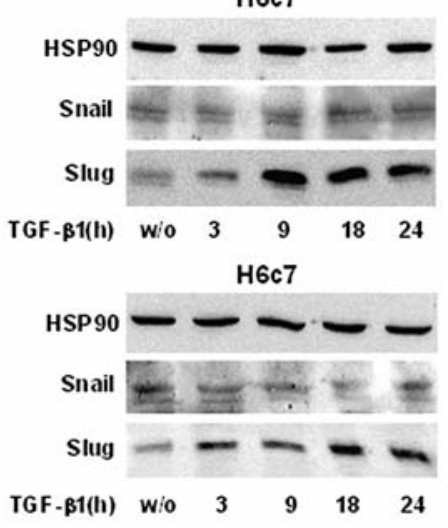

B)
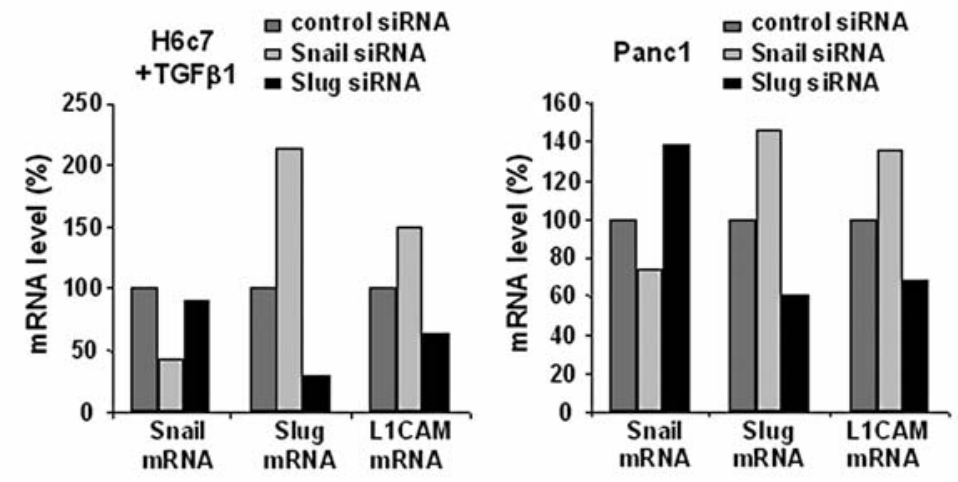

C)

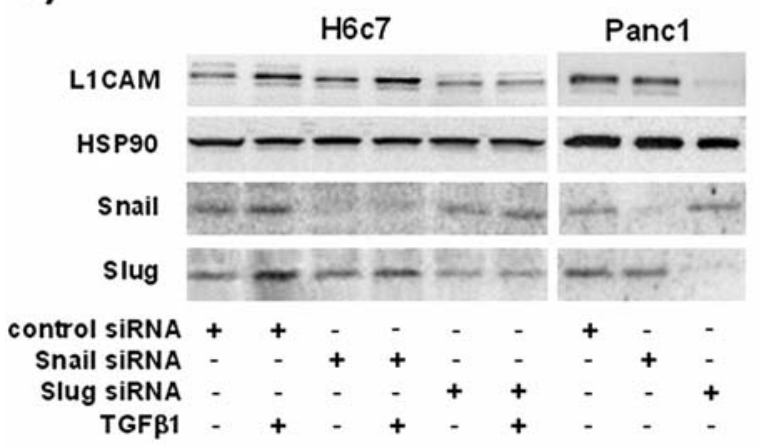

\section{D)}
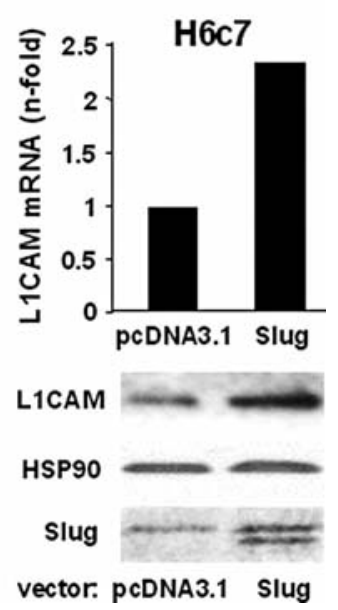

Figure 1. L1CAM expression in H6c7 and Panc1 cells is Slug dependent. (A) H6c7 cells were treated with $10 \mathrm{ng} / \mathrm{ml}$ TGF-B1 for the indicated periods. mRNA was submitted to real-time PCR for detection of Snail and Slug (left panel) using B-actin for normalization. Data from two independent experiments are shown. Nuclear extracts were submitted to Snail and Slug Western blots (right panel) using Hsp90 as control for equal protein loading, two independent experiments out of three are shown. (B) H6c7 cells treated with $10 \mathrm{ng} / \mathrm{ml}$ TGF-ß1 for $9 \mathrm{~h}$ and Panc1 cells, respectively, were treated with control, Snail or Slug siRNA. Then, mRNA was submitted to real-time PCR for detection of Snail, Slug and L1CAM. B-actin was used as control for normalization and data represent the mean of two independent experiments. (C) H6c7 cells treated with $10 \mathrm{ng} / \mathrm{ml}$ TGF- $\$ 1$ for 24 h or Panc1 cells were treated with control, Snail or Slug siRNA. Then, whole cell lysates were analysed by L1CAM, Snail and Slug Western blots, using Hsp90 as control for equal protein loading. A representative experiment out of three is shown. (D) H6c7 cells were transfected with recombinant Slug or the empty vector (pcDNA3.1). Expression of L1CAM was analysed by real-time PCR (upper panel) and Western blot analysis (lower panel) and Slug was analysed by Western blot (lower panel). Representative results from three independent experiments are shown in each panel.

diminished the L1CAM expression in TGF-ß1 treated H6c7 cells. In contrast, the siRNA-mediated knock-down of Snail did not result in a decrease of L1CAM expression in H6c7 cells. Next, we investigated the effect of Slug and Snail knock-down on L1CAM expression in the PDAC cell line Panc1 which is characterized by an autocrine TGF- $\$ 1$ respon- siveness along with a strong mesenchymal phenotype (31). As shown in Fig. 1B and C, L1CAM expression in Panc1 cells was similarly affected by the knock-down of Slug, but not by the knock-down of Snail. Thus, the inducing effect of TGF- $\beta 1$ on L1CAM expression in pancreatic ductal epithelial and PDAC cells depends on Slug. In support of 
this assumption, overexpression of recombinant Slug in H6c7 cells increased the expression of L1CAM on mRNA as well as on protein level (Fig. 1D).

The LICAM promoter includes two operating Slug binding sites. The finding that L1CAM expression is Slug dependent prompted us to explore the L1CAM promoter for the presence of potential binding sites for Slug. A sequence analysis identified two potential Slug binding sites located at position -1241 (pos. 40350, GeneBank accession U52112.2) and -433 (pos. 39542, GeneBank accession U52112.2) respectively, of the human L1CAM promoter (Fig. 2A). Both sites match the consensus E-box recognition motif CAGGTG/CACCTG (8). This putative promoter region precedes the distal transcriptional start site at pos. 39109 (GeneBank accession U52112.2) (30) giving rise of a transcript (32) that includes the exon-1 (108 bp, containing parts of the 5'UTR) and intron-1 (10119 bp). It has to be noted that the designation of this exon as exon-1 follows the numbering suggested by Kallunki et al (30), where exon-2 is the 184-bp exon (which is sometimes also referred to as exon-1) containing the start codon.

To verify usage of this transcriptional start site, an additional conventional PCR setting was chosen specifically detecting the exon-1/exon-2 containing L1CAM mRNA transcript. As shown in Fig. 2B, the expected 153-bp PCRproduct was detectable in Panc1 cells as well as in H6c7 cells. Slug siRNA treatment of Panc1 resulted in a decline in the amount of this mRNA (Fig. 2B, upper panel). Moreover, the rather low level of this transcript in $\mathrm{H} 6 \mathrm{c} 7$ cells compared to Panc1 cells increased upon Slug transfection or after TGF-ß1 treatment (Fig. 2B, upper panel). Confirming these data quantitatively, real-time PCR detected 5-fold higher levels of this transcript in untreated Panc1 cells than in untreated H6c7 cells (Fig. 2B, lower panel). Treatment of Panc1 cells with Slug siRNA reduced the amount of this transcript by $30-40 \%$, whereas a 2-3-fold higher level of this mRNA was detected in H6c7 cells after TGF-B1 treatment or Slug transfection (Fig. 2B, lower panel).

In order to demonstrate the binding of Slug to its two potential binding motifs, oligonucleotides corresponding to both recognition sites were radiolabeled and analysed in gel shift assays. Using nuclear extracts from HeLa cells transfected with recombinant Slug (Fig. 2C), the formation of nuclearprotein-complexes with both labeled oligonucleotides (Fig. 2D) was detectable, whereas no such signals were seen with pcDNA3.1-transfected HeLa cells. The specificity of the formed DNA-protein-complex was verified by using a Slug antibody, which produced a supershifted DNA-proteincomplex (Fig. 2D). Supporting the Slug dependency of L1CAM expression, Western blot analysis detected also an increased level of L1CAM in HeLa cells overexpressing Slug (Fig. 2C).

Slug binds to both sites of the L1CAM promoter in H6c7 and Pancl cells. In order to determine whether Slug is capable of direct DNA binding to both of the two potential binding sites, in vivo chromatin immunoprecipitation assays (ChIP) were performed using primers spanning either one of the Slug binding sites in the L1CAM promoter. Analysis of H6c7 cells was done after treatment with TGF- 11 for $9 \mathrm{~h}$, a period at which the highest level of nuclear Slug is reached in H6c7 cells (16). Under these conditions, the amplification signals of both binding sites obtained from anti-Slug immunoprecipitated DNA were significantly greater than those with DNA from untreated H6c7 cells (Fig. 3A). In contrast, the amplification signals of both sites from anti-Snail immunoprecipitated DNA declined after treatment with TGF- 31 (Fig. 3A).

In order to verify the specificity of the amplification signals from anti-Slug immunoprecipitated DNA, H6c7 cells were treated with Slug siRNA followed by TGF- $\beta 1$ treatment. Compared to control siRNA-treated H6c7 cells, both binding sites were detected in anti-Slug immunoprecipitated DNA at significantly lower levels if Slug expression was silenced (Fig. 3B). Similarly, ChIP assays revealed that specific DNA binding of Slug to both binding sites of the L1CAM promoter in Panc1 cells exhibiting autocrine TGF- 11 secretion was significantly reduced by knock-down of Slug expression (Fig. 3C)

Owing to the fact that RNA-polymerase II is commonly recruited to transcriptional active genomic regions, ChIP assays were also performed with RNA-polymerase II-specific antibodies. In TGF- 31 -stimulated H6c7 cells as well as in Panc1 cells, RNA-polymerase II binding to both binding sites of the L1CAM promoter was reduced by siRNA-mediated knock-down of Slug (Fig. 3B and C).

Binding of Slug to the LICAM promoter is able to induce promoter activation. In order to underline the ChIP assay results and to verify Slug-dependent activation of the L1CAM promoter, luciferase assays were conducted. Various promoter constructs containing both Slug binding sites (-1425[Slug1/2]) or mutations in one or both of these sites were fused to the firefly reporter gene (Fig. 4A). For normalization, cells were co-transfected with a renilla luciferase construct.

H6c7 cells transfected with recombinant Slug and the -1425[Slug1/2] promoter construct showed a clearly increased luciferase expression (Fig. 4A, upper panel) compared to H6c7 cells co-transfected with pcDNA3.1. In contrast, no such Slug-dependent increase of luciferase activity was seen in $\mathrm{H} 6 \mathrm{c} 7$ cells transfected with the promoterless luciferase construct.

Next, promoter constructs with mutated binding sites were investigated in Slug expressing H6c7 cells. When targeting the first Slug binding site $(-1241 / 1236)$ by two nucleotide

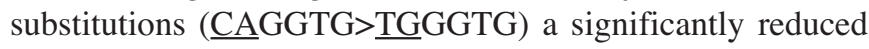
luciferase signal was obtained with the construct -1425 [Slug $\Delta 1 / 2$ ] (Fig. 4A, lower panel). A similar reducing effect on Slug-dependent luciferase expression was seen with the construct -1425 [Slug1/ $\Delta 2$ ] bearing a double mutation

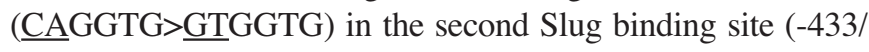
428). Finally, a construct exhibiting mutations in both Slug binding sites $(-1425[\operatorname{slug} \Delta 1 / \Delta 2])$ was analysed. In this case, the Slug-dependent reporter gene activity was completely abrogated in Slug-transfected H6c7 cells.

Compared to control siRNA treatment, Panc1 cells transfected with the -1425 [Slug1/2] promoter construct exhibited a decreased luciferase expression if treated with Slug siRNA (Fig. 4B, upper panel), thus in line with the Slug dependency 
A)

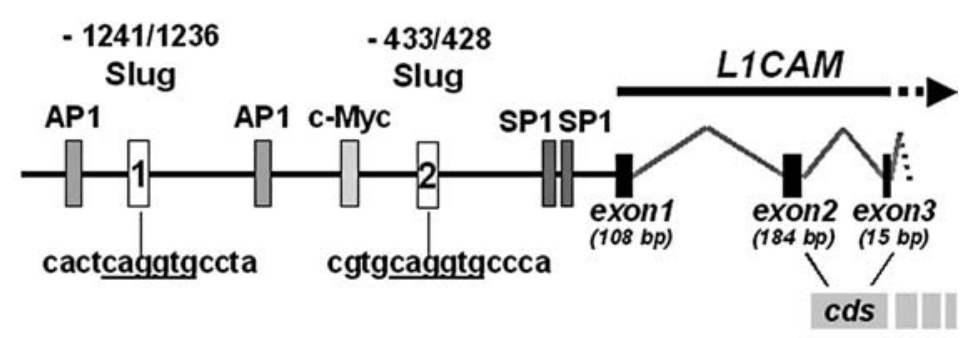

B)
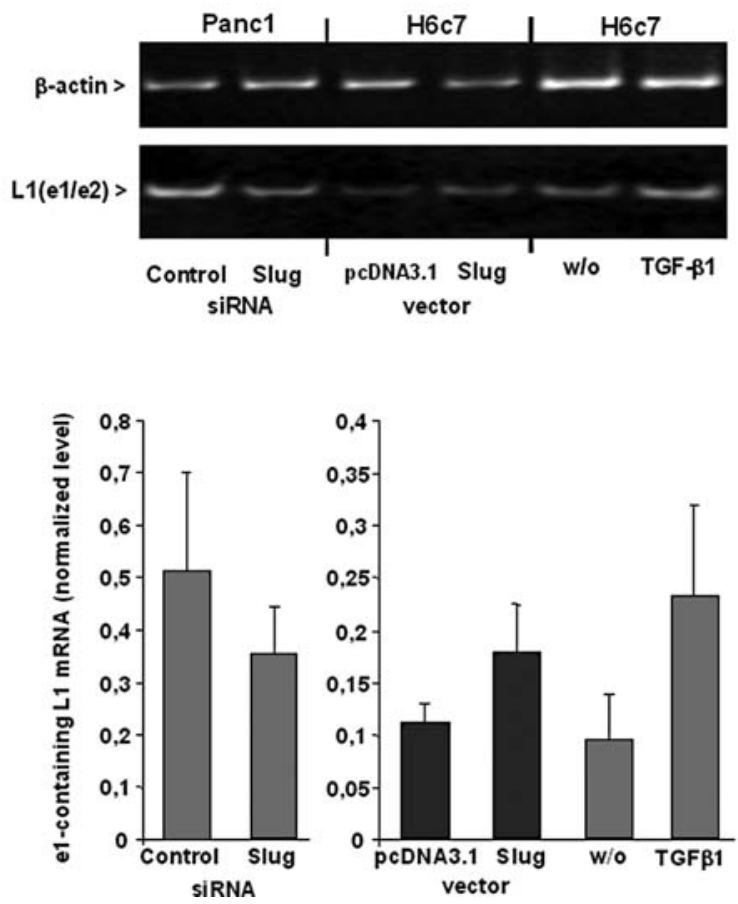

C)

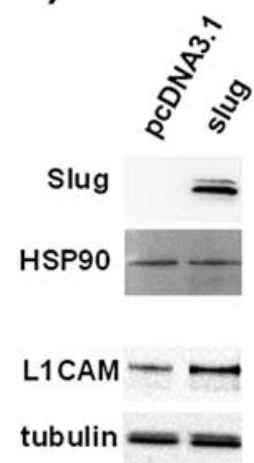

D)

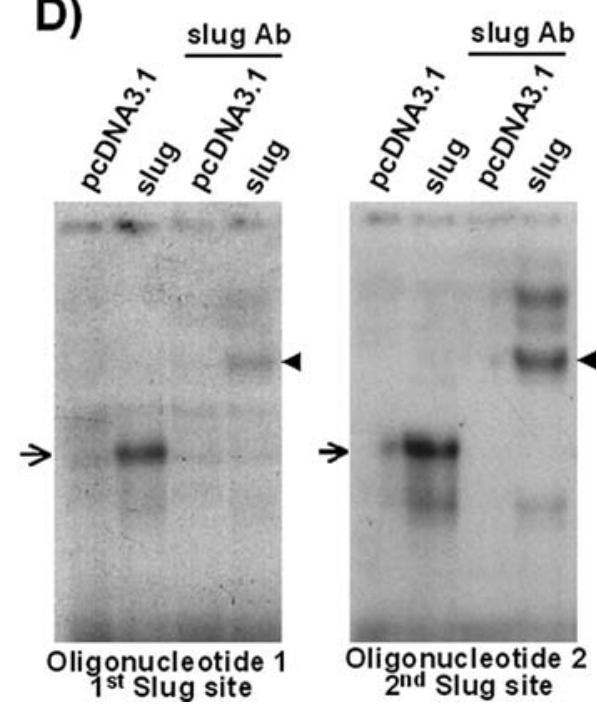

Figure 2. The L1CAM promoter comprises two Slug binding sites and Slug can bind to both sites. (A) By sequence analysis two recognition motifs for Slug binding were identified in the L1CAM promoter at positions $-1241 / 1236$ and $-433 / 428$. The consensus motif of the DNA sequences in these regions (written below) is underlined. The L1CAM primary transcript is indicated by the bold line and dashed arrow above the first three exons and introns (sizes not in scale), the coding strand (cds) is indicated below exon-2 and exon-3. (B) mRNA from Panc1 cells subject of control or Slug siRNA treatment and from H6c7 cells subject of Slug transfection or TGF- $\beta 1$ treatment $(10 \mathrm{ng} / \mathrm{ml}, 9 \mathrm{~h})$ was submitted to conventional RT-PCR for specific detection of the exon-1 containing L1CAM mRNA transcript [L1(e1/e2)]. B-actin PCR was used as control. In parallel, quantitative results were obtained from real-time PCR analysing the L1(e1/e2)-amplicon. B-actin was used as control for normalization and data represent the mean of three independent experiments. (C) Slug expression in nuclear extracts as well as L1CAM expression in whole cell lysates of HeLa cells transfected with recombinant Slug or pcDNA3.1 were shown by Western blot, using Hsp90 and tubulin, respectively, as loading control. (D) Nuclear extracts from Slug or pcDNA3.1-transfected HeLa cells were used in gel shift assays with ${ }^{32} \mathrm{P}$-labeled oligonucleotides containing one Slug site each. For specificity control, supershifts were performed using anti-Slug antibodies. One representative result from 2 independent experiments is shown. 


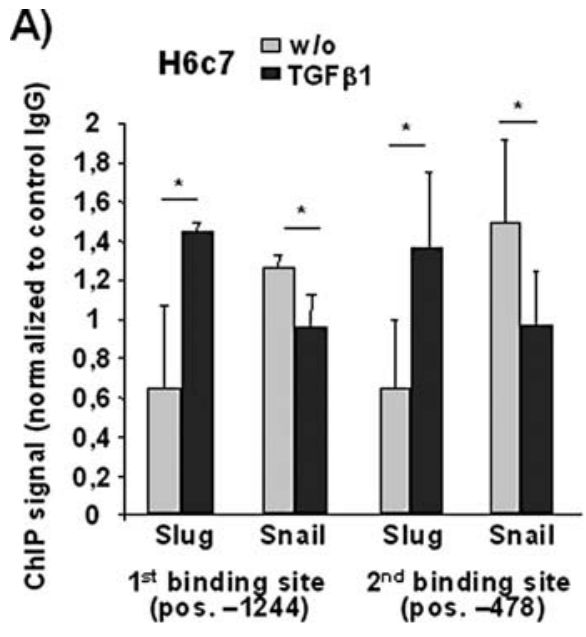

B)
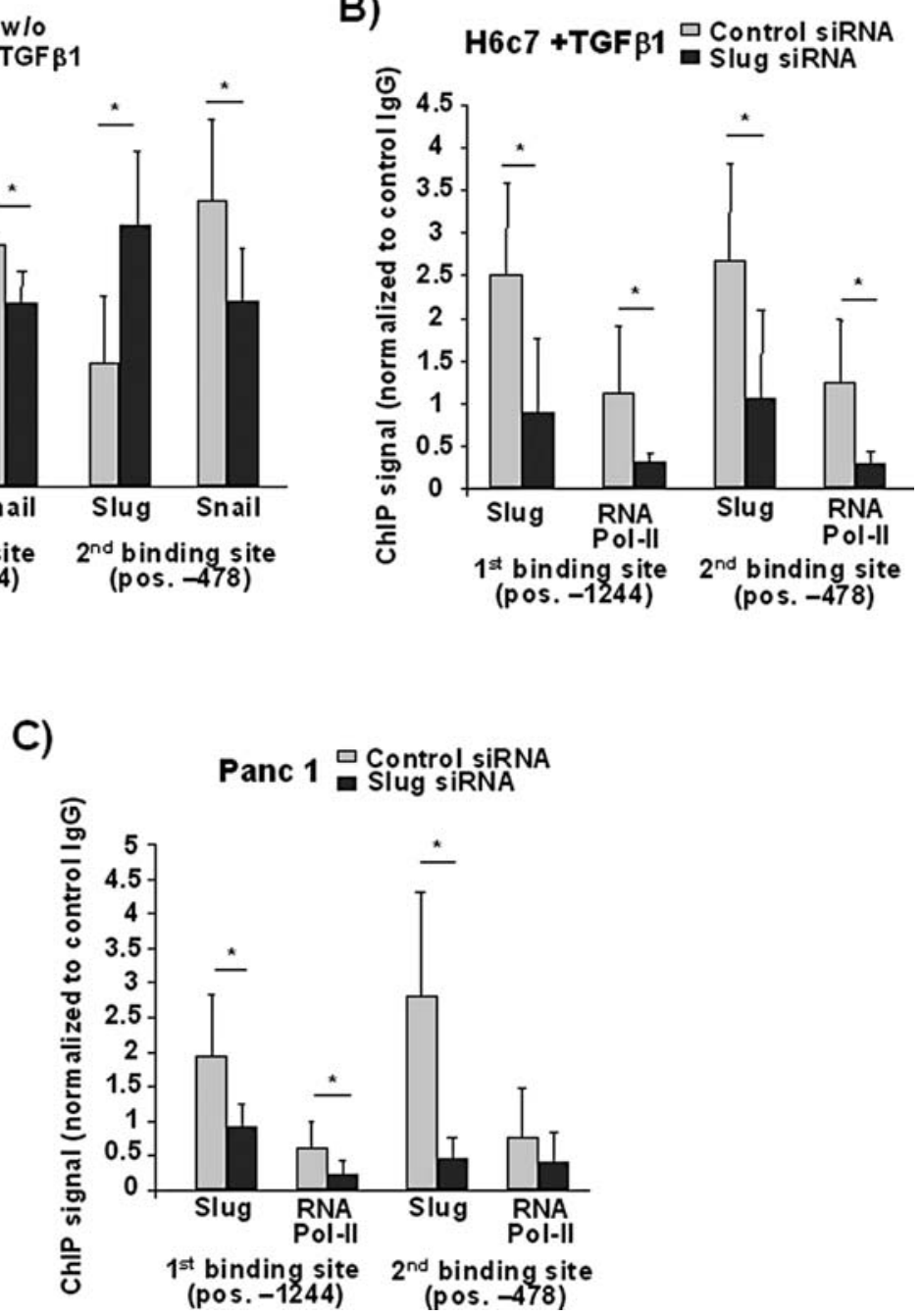

Figure 3. The transcription factor Slug binds to the L1CAM promoter in vivo. (A) H6c7 cells were treated for $9 \mathrm{~h}$ with TGF- 31 , and ChIP assays were conducted using Slug- and Snail-specific antibodies. Binding of Slug and Snail to the promoter was detected by real-time PCR. Upon normalization to GAPDH, results were expressed as n-fold compared to specific antibody controls. Data are expressed as mean \pm SD from four independent experiments; ${ }^{*} \mathrm{p}<0.05$. (B) H6c7 cells were transfected with control siRNA or Slug siRNA and then stimulated for $9 \mathrm{~h}$ with TGF-ß1. (C) Panc1 cells were transfected with control siRNA or Slug siRNA. (B and C) ChIP assays were conducted using Slug and RNA-polymerase II-specific antibodies. Binding of Slug and RNApolymerase II to the promoter was detected by real-time PCR. Upon normalization to GAPDH, results were expressed as n-fold compared to specific antibody controls. Data are expressed as mean $\pm \mathrm{SD}$ from four independent experiments; ${ }^{*} \mathrm{p}<0.05$.

of L1CAM expression in this cell line. Similar to the results with Slug-transfected H6c7 cells, Panc1 cells showed significantly reduced reporter gene activities when transfected with promoter constructs harbouring mutations in either one of the Slug sites (Fig. 4B) and, again, the promoter activity was almost abolished if both Slug binding sites had been mutated. By demonstrating that the transcription factor Slug binds to the L1CAM promoter in pancreatic ductal epithelial and PDAC cells along with an activation of gene transcription these results indicate that L1CAM is a novel target gene of Slug.

\section{Discussion}

The expression of the Snail transcription factor family is often associated with EMT during developmental processes as well as tumor progression (9,33-35). Besides the repression of E-cadherin $(8,10)$ which is a hallmark of EMT in cancer, other transcriptional targets are affected by the Slug/
Snail family proteins during EMT, as well. Amongst these, we recently identified the promigratory molecule L1CAM, which is strongly upregulated in human pancreatic ductal epithelial (HPDE/H6c7) cells during TGF-31-induced EMT (16). Interestingly, it was found that the strong inducing effect of TGF-B1 depends on Slug, but not on Snail.

Our present study revealed, that Slug directly induces L1CAM expression by an interaction with two specific binding sites located at position 433 and 1241 upstream of the most distal transcriptional start giving rise of a L1CAM transcript containing exon-1 and intron-1. This transcript is the product of a differentially recruited transcriptional starting point besides five others located within the first intron or even further downstream (32). Thus, in epithelial cells, L1CAM expression seems to be governed by the 5'-region upstream of exon-1. In its first part, this region is of high similarity with the murine L1CAM promoter, as already shown by Kallunki et al (30). However, the two Slug/Snail binding sites located 


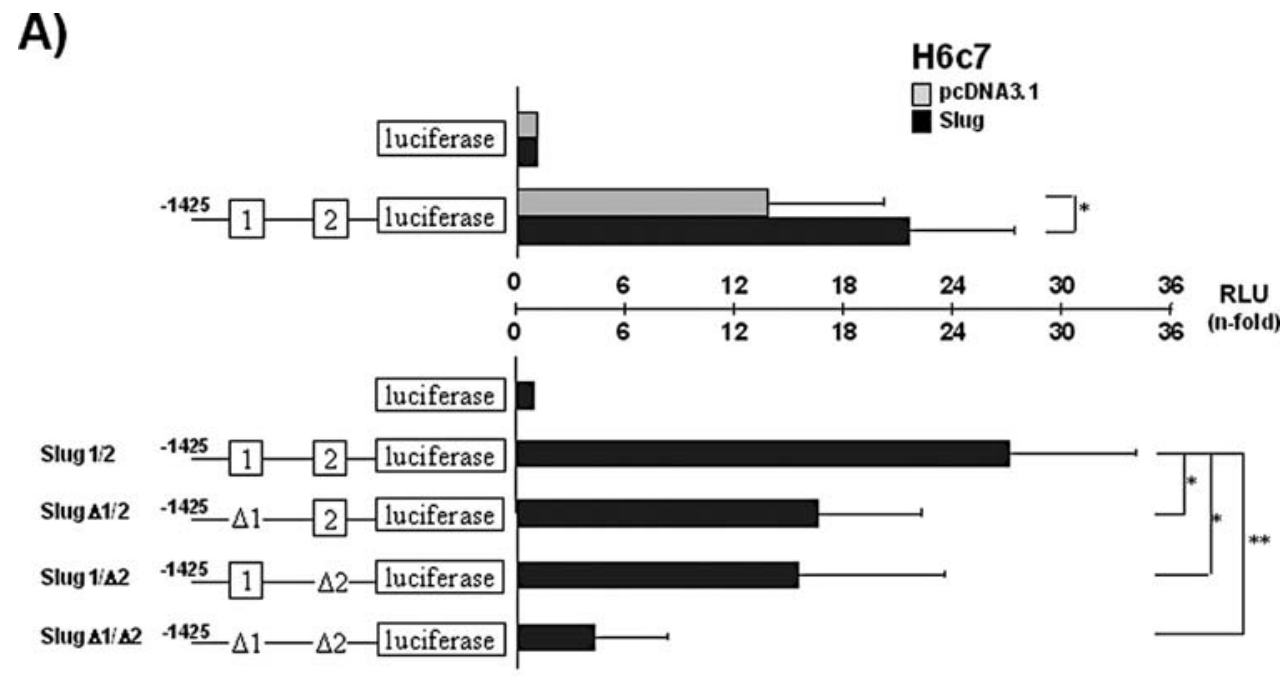

B)

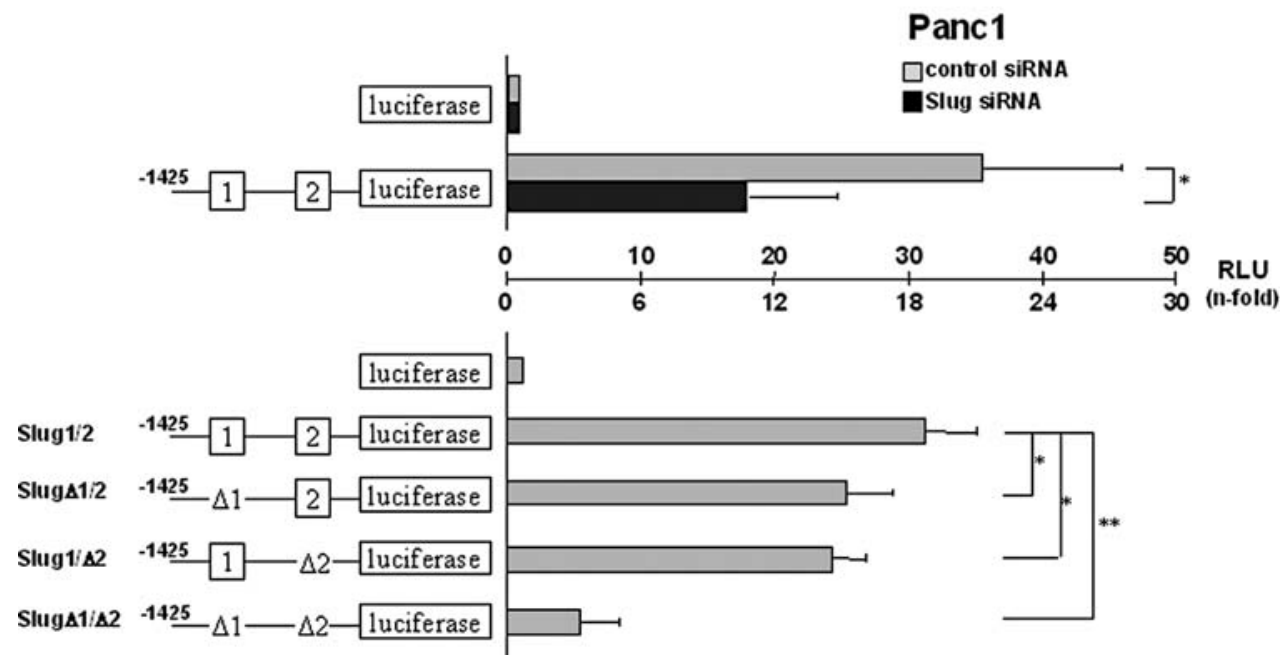

Figure 4. Transcriptional activation of L1CAM promoter constructs by Slug in H6c7 and Panc1 cells. (A) Slug or pcDNA3.1-transfected H6c7 cells were cotransfected with the 1425-bp L1CAM promoter-luciferase construct containing both Slug binding sites, or Slug-transfected H6c7 cells were co-transfected with the 1425-bp L1CAM promoter-luciferase construct containing both Slug binding sites (-1425[Slug1/2]) or with promoter constructs bearing mutations in either one (-1425[Slug $\Delta 1 / 2$ ], -1425 [slug1/ $\Delta 2$ ]) or in both Slug binding sites $(-1425$ [Slug $\Delta 1 / \Delta 2$ ]). (B) Slug siRNA or control siRNA-transfected Panc1 cells were co-transfected with the 1425-bp L1CAM luciferase construct or control siRNA-transfected Panc1 cells were co-transfected with the L1CAM promoter variants. (A and B) Firefly luciferase expression was normalized to renilla luciferase expression. The bar graph indicates the mean fold induction \pm s.d. from at least four independent experiments; ${ }^{*} \mathrm{p}<0.05,{ }^{* *} \mathrm{p}<0.01$.

at positions -433 and -1241 are exclusively found in the human L1CAM promoter.

Both sites interact with Slug during TGF- 11 treatment of H6c7 cells as shown by ChIP assays. Since this interaction was not seen before $9 \mathrm{~h}$ (data not shown) it can be assumed that the Slug-dependent effect of TGF- $B 1$ on L1CAM expression is preceded by an induction of Slug expression by TGF- $\beta 1$ which indeed was maximal within 3-9 h of TGF- 31 treatment (Fig. 1A). Besides its increased de novo expression, post-translational modifications of Slug may also occur later on after TGF- $\$ 1$ administration accounting for the even more pronounced rise in the nuclear level of the Slug protein noted after $9 \mathrm{~h}$ (Fig. 1A) and the somewhat delayed peak (16-24 h) of L1CAM expression.
In contrast, Snail expression remained unaffected or even slightly declined in H6c7 cells subject of TGF-ß1 treatment. Moreover, binding of Snail, which potentially interacts with the same consensus sequence, to both binding sites was reduced in $\mathrm{H6c7}$ cells treated with TGF- 31 . One can speculate that resulting from its TGF- 31 -induced expression and forced nuclear accumulation Slug displaces Snail from the L1CAM promoter. This Slug/Snail displacement then accounts for transcriptional activation, perhaps via the release of the L1CAM promoter from Snail-dependent repression. In support of this assumption, knock-down of Snail slightly increases L1CAM expression in H6c7 cells, as can be appreciated from Fig. 1B and C. Another observation from these experiments was a slightly increased Snail expression in 
Panc1 cells subject of Slug knock-down, and vice versa an upregulation of Slug expression in $\mathrm{H6c} 7$ and Panc1 cells subject of Snail knock-down. This indicates a mutual modulation of Slug and Snail expression in these cell lines which may add to the Slug-dependent effect of TGF-B1 on L1CAM expression.

Furthermore, Slug-dependent recruitment of RNA-polymerase II to both binding sites (Fig. 3B and C) is indicative of a transcriptional activation of the human L1CAM promoter by Slug via both of these sites. A cooperative action by these two binding sites was demonstrated by luciferase assays, revealing that mutation of either one of these sites partially reduced and mutation of both sites completely abrogated the Slug-induced transcriptional activity of the human L1CAM promoter. Thus, our data indicate that Slug directly drives L1CAM expression via binding to two recognition sites in the L1CAM promoter during TGF-ß1-induced EMT of H6c7 cells. The involvement of Slug in this process was underscored by the finding that a similar Slug-dependent regulation of the L1CAM promoter was seen with the PDAC cell line Panc1. Since this cell line is characterized by a pronounced mesenchymal phenotype (31) along with high expression of L1CAM (16) and since these properties can be attributed to an autocrine secretion of TGF- $\beta 1(16,36)$, Panc1 cells could be regarded as being reminiscent of the effects of TGF- 11 seen in H6c7 cells.

An interesting recent study (37) also demonstrated the involvement of L1CAM expression in the EMT of Panc1 cells that was shown to be inversely related to the Syk tyrosine kinase and to be independent of $\beta$-catenin. Instead, the authors speculated an involvement of Slug/Snail in EMTassociated L1CAM expression in PDAC cells. Similarly in line with our findings, endometrial carcinoma (EC) cells also show a Slug-dependent and $\beta$-catenin-independent upregulation of L1CAM after TGF- 31 stimulation (38), and a recent study identified L1CAM as transcriptional target of Slug, but not of Snail in the epithelial ovarian cancer cell line A4 (39). Since H6c 7 cells already adopted a migratory and invasive phenotype under EMT inducing conditions and since the upregulation of L1CAM preceded E-cadherin repression, the Slug-dependent induction of L1CAM expression seems to be involved early in the EMT of PDAC. In contrast to the B-catenin-independent L1CAM expression in H6c7 and Panc1 cells [(29), data not shown], L1CAM expression has been shown to be induced via the $\beta$-catenin/Lef1 pathway in other tumor entities; e.g. in colon and breast cancer $(21,40)$. One explanation might be that the tumoral context, e.g. governed by epigenetic effects, defines whether L1CAM expression during EMT is induced through the Snail/E-cadherin/ß-catenin pathway (as in certain tumors such as colon cancer), thus being indirectly Snail-dependent, or through the direct transcriptional induction by Slug (as in other tumors such as PDAC and EC). Alternatively, the Slug and Snail/E-cadherin/ B-catenin-mediated L1CAM induction represent two consecutive events, the latter still being absent in H6c7 cells. Given the involvement of L1CAM in EMT related processes, its expression not only in higher grade pancreatic intraepithelial neoplasias (PanINs) and PDAC, but also in chronically inflamed pancreatic tissue (41) points to a novel mechanism by which Slug contributes to both the early onset of EMT during desmoplasia and further dedifferentiation processes during tumorigenesis of the pancreas.

\section{Acknowledgements}

The authors thank Maike Witt and Dagmar Leisner for technical support, Dr Antonio Garcia de Herreros (Universitat Pompeu Fabra, Barcelona, Spain) for helpful discussions and the German Research Society (DFG: Scha 677/7-3 and DFG: Se 1831/2-1) and the German Cluster of Excellence 'Inflammation at Interfaces' for funding.

\section{References}

1. Jemal A, Siegel R, Ward E, Murray T, Xu J and Thun MJ: Cancer statistics, 2007. CA Cancer J Clin 57: 43-66, 2007.

2. Polyak K and Weinberg RA: Transitions between epithelial and mesenchymal states: acquisition of malignant and stem cell traits. Nat Rev Cancer 9: 265-273, 2009.

3. Huber MA, Kraut $\mathrm{N}$ and Beug $\mathrm{H}$ : Molecular requirements for epithelial-mesenchymal transition during tumor progression. Curr Opin Cell Biol 17: 548-558, 2005.

4. Kabashima A, Higuchi H, Takaishi H, et al: Side population of pancreatic cancer cells predominates in TGF-beta-mediated epithelial to mesenchymal transition and invasion. Int J Cancer 124: 2771-2779, 2009.

5. Moustakas A and Heldin CH: Signaling networks guiding epithelial-mesenchymal transitions during embryogenesis and cancer progression. Cancer Sci 98: 1512-1520, 2007.

6. Cui W, Fowlis DJ, Bryson S, et al: TGFbeta1 inhibits the formation of benign skin tumors, but enhances progression to invasive spindle carcinomas in transgenic mice. Cell 86: 531-542, 1996.

7. Ellenrieder V, Hendler SF, Boeck W, et al: Transforming growth factor beta1 treatment leads to an epithelial-mesenchymal transdifferentiation of pancreatic cancer cells requiring extracellular signal-regulated kinase 2 activation. Cancer Res 61: 4222-4228, 2001.

8. Hemavathy K, Ashraf SI and Ip YT: Snail/slug family of repressors: slowly going into the fast lane of development and cancer. Gene 257: 1-12, 2000.

9. Alves CC, Carneiro F, Hoefler H and Becker KF: Role of the epithelial-mesenchymal transition regulator Slug in primary human cancers. Front Biosci 14: 3035-3050, 2009.

10. Batlle E, Sancho E, Franci C, et al: The transcription factor snail is a repressor of E-cadherin gene expression in epithelial tumour cells. Nat Cell Biol 2: 84-89, 2000.

11. Cano A, Perez-Moreno MA, Rodrigo I, et al: The transcription factor snail controls epithelial-mesenchymal transitions by repressing E-cadherin expression. Nat Cell Biol 2: 76-83, 2000.

12. Zavadil J and Bottinger EP: TGF-beta and epithelial-tomesenchymal transitions. Oncogene 24: 5764-5774, 2005.

13. De Craene B, Gilbert B, Stove C, Bruyneel E, van Roy F and Berx G: The transcription factor snail induces tumor cell invasion through modulation of the epithelial cell differentiation program. Cancer Res 65: 6237-6244, 2005.

14. Barrallo-Gimeno A and Nieto MA: The Snail genes as inducers of cell movement and survival: implications in development and cancer. Development 132: 3151-3161, 2005.

15. Hotz B, Arndt M, Dullat S, Bhargava S, Buhr HJ and Hotz HG: Epithelial to mesenchymal transition: expression of the regulators snail, slug, and twist in pancreatic cancer. Clin Cancer Res 13: 4769-4776, 2007.

16. Geismann C, Morscheck M, Koch D, et al: Up-regulation of L1CAM in pancreatic duct cells is transforming growth factor beta1- and slug-dependent: role in malignant transformation of pancreatic cancer. Cancer Res 69: 4517-4526, 2009.

17. Moos M, Tacke R, Scherer H, Teplow D, Fruh K and Schachner M: Neural adhesion molecule L1 as a member of the immunoglobulin superfamily with binding domains similar to fibronectin. Nature 334: 701-703, 1988.

18. Maness PF and Schachner M: Neural recognition molecules of the immunoglobulin superfamily: signaling transducers of axon guidance and neuronal migration. Nat Neurosci 10: 19-26, 2007.

19. Weidle UH, Eggle D and Klostermann S: L1-CAM as a target for treatment of cancer with monoclonal antibodies. Anticancer Res 29: 4919-4931, 2009. 
20. Fogel M, Mechtersheimer S, Huszar M, et al: L1 adhesion molecule (CD 171) in development and progression of human malignant melanoma. Cancer Lett 189: 237-247, 2003.

21. Gavert N, Conacci-Sorrell M, Gast D, et al: L1, a novel target of beta-catenin signaling, transforms cells and is expressed at the invasive front of colon cancers. J Cell Biol 168: 633-642, 2005.

22. Sebens Muerkoster S, Werbing V, Sipos B, et al: Drug-induced expression of the cellular adhesion molecule L1CAM confers anti-apoptotic protection and chemoresistance in pancreatic ductal adenocarcinoma cells. Oncogene 26: 2759-2768, 2007.

23. Boo YJ, Park JM, Kim J, et al: L1 expression as a marker for poor prognosis, tumor progression, and short survival in patients with colorectal cancer. Ann Surg Oncol 14: 1703-1711, 2007.

24. Fogel M, Gutwein P, Mechtersheimer S, et al: L1 expression as a predictor of progression and survival in patients with uterine and ovarian carcinomas. Lancet 362: 869-875, 2003.

25. Kaifi JT, Reichelt U, Quaas A, et al: L1 is associated with micrometastatic spread and poor outcome in colorectal cancer. Mod Pathol 20: 1183-1190, 2007.

26. Rawnaq T, Kleinhans H, Uto M, et al: Subset of esophageal adenocarcinoma expresses adhesion molecule 11 in contrast to squamous cell carcinoma. Anticancer Res 29: 1195-1199, 2009.

27. Furukawa T, Duguid WP, Rosenberg L, Viallet J, Galloway DA and Tsao MS: Long-term culture and immortalization of epithelial cells from normal adult human pancreatic ducts transfected by the E6E7 gene of human papilloma virus 16. Am J Pathol 148 : 1763-1770, 1996.

28. Muerkoster SS, Werbing V, Koch D, et al: Role of myofibroblasts in innate chemoresistance of pancreatic carcinoma-epigenetic downregulation of caspases. Int J Cancer 123: 1751-1760, 2008.

29. Sebens Muerkoster S, Kotteritzsch J, Geismann C, et al: alpha5integrin is crucial for L1CAM-mediated chemoresistance in pancreatic adenocarcinoma. Int J Oncol 34: 243-253, 2009.

30. Kallunki P, Edelman GM and Jones FS: Tissue-specific expression of the L1 cell adhesion molecule is modulated by the neural restrictive silencer element. J Cell Biol 138: 1343-1354, 1997.

31. Sipos B, Moser S, Kalthoff H, Torok V, Lohr M and Kloppel G: A comprehensive characterization of pancreatic ductal carcinoma cell lines: towards the establishment of an in vitro research platform. Virchows Arch 442: 444-452, 2003.
32. Kimura K, Wakamatsu A, Suzuki Y, et al: Diversification of transcriptional modulation: large-scale identification and characterization of putative alternative promoters of human genes. Genome Res 16: 55-65, 2006.

33. Jethwa P, Naqvi M, Hardy RG, et al: Overexpression of Slug is associated with malignant progression of esophageal adenocarcinoma. World J Gastroenterol 14: 1044-1052, 2008.

34. Medici D, Hay ED and Goodenough DA: Cooperation between snail and LEF-1 transcription factors is essential for TGF-beta1induced epithelial-mesenchymal transition. Mol Biol Cell 17: 1871-1879, 2006.

35. Peinado H, Quintanilla $\mathrm{M}$ and Cano A: Transforming growth factor beta-1 induces snail transcription factor in epithelial cell lines: mechanisms for epithelial mesenchymal transitions. J Biol Chem 278: 21113-21123, 2003.

36. Chen WB, Lenschow W, Tiede K, Fischer JW, Kalthoff $H$ and Ungefroren H: Smad4/DPC4-dependent regulation of biglycan gene expression by transforming growth factor-beta in pancreatic tumor cells. J Biol Chem 277: 36118-36128, 2002.

37. Layton T, Stalens C, Gunderson F, Goodison S and Silletti S: Syk tyrosine kinase acts as a pancreatic adenocarcinoma tumor suppressor by regulating cellular growth and invasion. Am J Pathol 175: 2625-2636, 2009.

38. Huszar M, Pfeifer M, Schirmer U, et al: Up-regulation of L1CAM is linked to loss of hormone receptors and E-cadherin in aggressive subtypes of endometrial carcinomas. J Pathol (In press).

39. Kurrey NK, Jalgaonkar SP, Joglekar AV, et al: Snail and slug mediate radioresistance and chemoresistance by antagonizing p53-mediated apoptosis and acquiring a stem-like phenotype in ovarian cancer cells. Stem Cells 27: 2059-2068, 2009.

40. Shtutman M, Levina E, Ohouo P, Baig M and Roninson IB: Cell adhesion molecule L1 disrupts E-cadherin-containing adherens junctions and increases scattering and motility of MCF7 breast carcinoma cells. Cancer Res 66: 11370-11380, 2006.

41. Bergmann F, Wandschneider F, Sipos B, et al: Elevated L1CAM expression in precursor lesions and primary and metastastic tissues of pancreatic ductal adenocarinoma. Oncol Rep 24: 909915, 2010. 